\title{
Throughput Analysis of Unslotted CSMA with Grouped Sensing in Multi-Channel Cognitive Radio Network
}

\author{
Hongqian Fang ${ }^{1, a}$ and Dongbi Zhu ${ }^{\star 1, b}$ \\ ${ }^{1}$ Department of Electronics and Communication Engineering, \\ College of Engineering, Yanbian University, Yanji, China \\ afanghongqian@jl.chinamobile.com, bdbzhu@ybu.edu.cn \\ ${ }^{*}$ corresponding author
}

Keywords: cognitive radio network; CSMA; grouped sensing; throughput; HoL packet delay

\begin{abstract}
We propose an unslotted CSMA with grouped sensing and analyze the throughput of SUs in multi-channel cognitive radio network. We model the system by 3-demensional continuous time Markov process, calculate the steady state probability of the system, and obtain throughput and transmission delay of SUs. By comparing the performances of grouped sensing scheme, random $m$-sensing and hybrid sensing, the numerical results show that grouped sening scheme overcome the defect of low throughput in random $m$-sensing scheme.
\end{abstract}

\section{Introduction}

With the rapid development of wireless communication technique, the conflict between limited spectrum resource and the increasing requirements for wireless access has become the main contradiction in future wireless communication. The FCC has reported that the licensed bands are vastly underutilized [1]. Cognitive radio networks have recently emerged as a promising technique to improve the utilization of the existing radio spectrum. Cognitive radio provides high spectrum efficiencies by enabling secondary users(SUs) to use the channels which are not used by primary users(PUs).

There have been many studies on the opportunistic spectrum access for cognitive radio network. We classify the opportunistic spectrum access as the centralized (e.g., [2,3]) and decentralized(e.g., [4-10]) cognitive medium access control (MAC) protocols. In decentralized MAC protocols, SUs individually perform spectrum sensing and spectrum scheduling. The decentralized cognitive MAC protocols can be further classified as slotted structure (e.g., [4-6]) and unslotted structure (e. g., [7-10]) where the slotted structure means the time axis for network is divided into time slots and all PUs and SUs are synchronous.

S. Huang et. al. [7] proposed three decentralized MAC protocols with different sensing and backoff mechanisms. D. B. ZHU et. al. [8] analyzed the performance of random $m$-sensing scheme in unslotted cognitive radio network. They obtained that the throughput of SUs in unslotted CSMA with random $m$-sensing increases as the number $m$ of sensing channels increases, and the throughput of SUs with all sensing is much larger than that with random sensing. But SU must senses all channels before transmitting each packet in all sensing scheme, the power consumption of SUs' terminal devices will be very high. Oppositely, the power consumption of SUs' terminal devices is low in random $m$-sensing scheme when $m$ is small, but the performance is poor. How to solve the contradiction between throughput and power consumption is the motivation of our work.

In this paper, we propose and analyze unslotted CSMA with grouped sensing, which senses channels based on grouping. This grouped sensing scheme can overcome the defects of low throughput in random $m$-sensing and high power consumption of SUs' terminal devices in all sensing.

The rest of this paper is organized as follows: In section 2, the system model of unslotted CSMA with grouped sensing is described in detail. In section 3, the performance of unslotted CSMA with 
grouped sensing is analyzed. Numerical examples are presented in section 4 and summary is given in section 5 .

\section{System Model}

We consider an unslotted CSMA in multi-channel cognitive radio network with $N$ SUs and $M$ channels. Channels are divided into 2 groups, $A$ and $B$, group $A$ includes $m$ channels and group $B$ includes the other $M-m$ channels. The PU's usage pattern of a channel follows On/Off process and is independent with other's channel usage patterns. An On-period is the time duration in which the PU occupies the channel, an Off-period is the time duration in which the PU does not occupy the channel and the SUs can opportunistically use the channel. It is assumed that the On-periods and Off-periods on the channel have exponential distribution with mean $\alpha^{-1}$ and $\beta^{-1}$ respectively. Because of the primary network and secondary network are unslotted structure, time synchronization among SUs and PUs are not needed.

We assume that all SUs are saturated, which means each SU always has packet to transmit. And each SU performs perfect sensing (i.e., no false alarm and no mis-detection) and the sensing period of channels is negligible.

The unslotted CSMA with grouped sensing is operated as follows:

At the beginning, each SU senses channels from group A, before its packet transmitted.

1) If an SU finds idle channels from group $A$, the SU selects one idle channel randomly and transmits a packet, then the SU goes to backoff state after packet transmission, in order to prevent the channel being exclusively used by specific SU.

2) If the SU does not find any idle channel from group $A$, then the SU goes to backoff state and senses the channels from group $B$ after its random backoff time.

- When the SU finds idle channels from group $B$, the SU selects one idle channel randomly and transmit a packet. After packet transmission the SU goes to backoff state and access the system by procedure 1) after its backoff time.

- When the SU does not find any idle channel from group $B$, the SU goes to backoff state immediately, and access the system by procedure 1) after its backoff time.

3) When a PU returns to the channel on which an SU is transmitting a packet, the SU must vacate the channel to the PU and sense all channels. If idle channels are found, the SU selects one of idle channels to perform spectrum handoff, otherwise the SU gives up its packet transmission and goes to backoff state, then performs above processes repeatedly.

We assume the SU's packet transmission time is exponentially distributed with mean $\mu^{-1}$, the SUs' backoff times are i.i.d, and exponentially distributed with mean $v^{-1}$.

\section{Performance Analysis}

Let

$N_{p}(t)=$ the number of channels transmitting PU's packet at time $t$;

$N_{s 1}(t)=$ the number of channels transmitting SU's packet at time $t$;

$N_{s 2}(t)=$ the number of SUs needing to sense channels from $B$ at time $t$.

Then $\left\{\left(N_{p}(\mathrm{t}), N_{s 1}(\mathrm{t}), N_{s 2}(\mathrm{t})\right) \mid \mathrm{t} \geq 0\right\}$ forms 3-dimensional Markov process with state space $S=\{(i, j, k) \mid 0 \leq i \leq M, 0 \leq j \leq M-i, 0 \leq k \leq N-j\}$.

Since the process $\left\{\left(N_{p}(\mathrm{t}), N_{s 1}(\mathrm{t}), N_{s 2}(\mathrm{t})\right) \mid \mathrm{t} \geq 0\right\}$ is an irreducible Markov process with finite state space, it is always ergodic and exists the steady state probability. Let $\pi_{i, j, k}$ is the joint probability that the Markov process is in state $(i, j, k)$, then we can obtain the following balance equantions:

$$
\begin{aligned}
& {[(N-j) v+(M-i) \beta+i \alpha+j \mu] \pi_{i, j, k}=(M-i+1) \beta \pi_{i-1, j, k}+(i+1) \alpha \pi_{i+1, j, k}} \\
& +(j+1) \mu \pi_{i, j+1, k}+(k+1) v g_{i, j} \pi_{i, j, k+1}+(k+1) v\left(1-g_{i, j}\right) \pi_{i, j-1, k+1}+(N-j-k+1) v q_{i, j} \pi_{i, j, k-1} \\
& +(N-j-k+1) v\left(1-q_{i, j}\right) \pi_{i, j-1, k}, \quad \text { for } 0 \leq i \leq M, 0 \leq j \leq M-i, 0 \leq k \leq N-j
\end{aligned}
$$


where $q_{i, j}\left(g_{i, j}\right)$ is the probability that all $m(M-m)$ channels in group $A(B)$ are busy when there are $i+j$ busy channels among $M$ channels, i.e.,

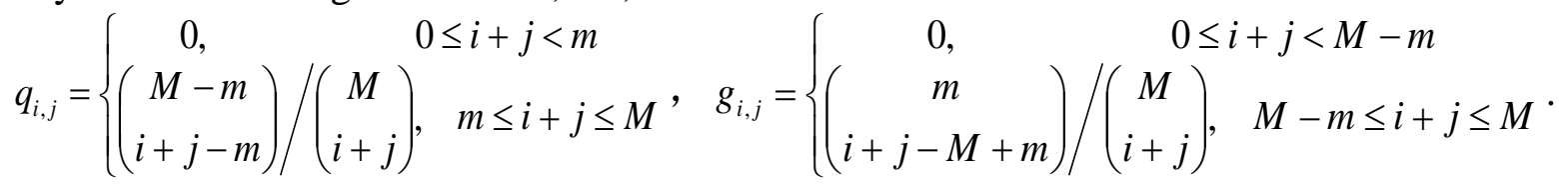

Note that $\left(\begin{array}{l}n \\ m\end{array}\right)=\frac{n !}{m !(n-m) !}$ and $\pi_{i, j, k}=0$ when $(i, j, k) \notin S$.

Since the initial condition of probability is given by

$$
\sum_{i=0}^{M} \sum_{j=0}^{M-i} \sum_{k=0}^{N-j} \pi_{i, j, k}=1 \text {. }
$$

By solving the linear equations (1) and (2), we can calculate the steady state probability $\pi_{i, j, k}$, and so we can obtain three important performance measures of the system.

1) Normalized throughput of SUs

The normalized throughput $T_{s}$ of SUs per channel is defined as the mean number of successfully transmitted packets during a service time of SU's packet on a channel.

To find $T_{s}$, let $\lambda_{e}$ be the effective packet arrival rate of SUs in the system and $\tilde{\pi}_{i, j}$ be the arrival point probability that he SU finds the system in the state $(i, j)$ upon sensing after its backoff time.

The effective packet arrival rate $\lambda_{e}$ is given by

$$
\lambda_{e}=\lambda_{e 1}+\lambda_{e 2}=\sum_{i=0}^{M} \sum_{j=0}^{M-i} \sum_{k=0}^{N-j}(N-j-k) v \pi_{i, j, k}+\sum_{i=0}^{M} \sum_{j=0}^{M-i} \sum_{k=0}^{N-j} k v \pi_{i, j, k}
$$

where $\lambda_{e 1}$ and $\lambda_{e 2}$ are the effective packet arrival rates of SUs who will sense channels from $A$ and $B$, respectively.

Hence, the arrival point probability $\tilde{\pi}_{i, j}$ can be given by

$$
\tilde{\pi}_{i, j}=\frac{\sum_{k=0}^{N-j-1}(N-j-k) v \pi_{i, j, k}}{\lambda_{e}}+\frac{\sum_{k=0}^{N-j-1} k v \pi_{i, j, k}}{\lambda_{e}}=\lambda_{e}^{-1} \sum_{k=0}^{N-j-1}(N-j) v \pi_{i, j, k}
$$

Let $P_{b}$ be the probability that the SU's packet is blocked due to no idle channels in the system upon sensing after its backoff time, then we have

$$
P_{b}=\frac{\lambda_{e 1}}{\lambda_{e}} \sum_{i=0}^{M} \sum_{j=0}^{M-i} \tilde{\pi}_{i, j} q_{i, j}+\frac{\lambda_{e 2}}{\lambda_{e}} \sum_{i=0}^{M} \sum_{j=0}^{M-i} \tilde{\pi}_{i, j} g_{i, j}
$$

During an SU transmitting a packet on a channel, it may be interrupted by PUs. The interrupted SU senses the channels again by all sensing scheme and then either continues to its packet transmission if the SU finds idle channels or goes to backoff state resulting in unsuccessful packet transmission. Let $P_{f}$ be the probability that the SU does not find any idle channels when the SU interrupted by a PU and senses the channels, then we have $P_{f}$

$$
P_{f}=\sum_{\{(i, j) \mid i \geq 1, i+j=M\}} \tilde{\pi}_{i, j} / \sum_{\{(i, j) \mid i \geq 1, i+j \leq M\}} \tilde{\pi}_{i, j}
$$

Since SU's packet may be forced to termination after a number of interruptions by PUs, we can obtain the forced termination probability $P_{F}$ of SUs as follows:

$$
P_{F}=\frac{\beta P_{f}}{\mu+\beta P_{f}} .
$$

By the definition of throughput $T_{s}$ of SUs, we have

$T_{s}=\left(1-P_{b}\right)\left(1-P_{F}\right) \lambda_{e} \mu^{-1} / M$.

2) Head-of-Line(HoL) packet delay of $\mathrm{SU}$ 
The HoL packet delay D of SU is defined by the time period from the time of reaching to head of buffer to the beginning point of the packet transmission. Each SU goes to backoff state after packet transmission and then senses the channels again after its backoff time. If the SU does not find any idle channels by its sensing, then the SU goes to backoff state again. After sensing, the SU goes to backoff state with probability $P_{\mathrm{b}}$ and transmits the packet with probability $1-P_{\mathrm{b}}$. Since the number of backoff state due to no idle channels has geometric distribution with parameter $1-P_{\mathrm{b}}$, and the backoff time is exponential distributed with rate $v$, so the HoL packet delay $D$ also is geometric sum of exponential random variables. Thus $D$ has exponential distribution with rate $\left(1-P_{\mathrm{b}}\right) v$ and the mean of HoL packet delay is given by

$$
E[D]=\left[\left(1-P_{b}\right) v\right]^{-1} \text {. }
$$

\section{Numerical Examples}

In this section, we present numerical examples for the performance measures of SUs . We assume there are $M=10$ channels in the system and the mean packet transmission time $\mu^{-1}=5$ msec. We set the parameter $\beta^{-1}=0.65 \mathrm{sec}$ and $\alpha^{-1}=0.35 \mathrm{sec}$, so that the channel idle probability is 0.65 . Let the mean backoff time of SUs $v^{-1}=20$ msec.
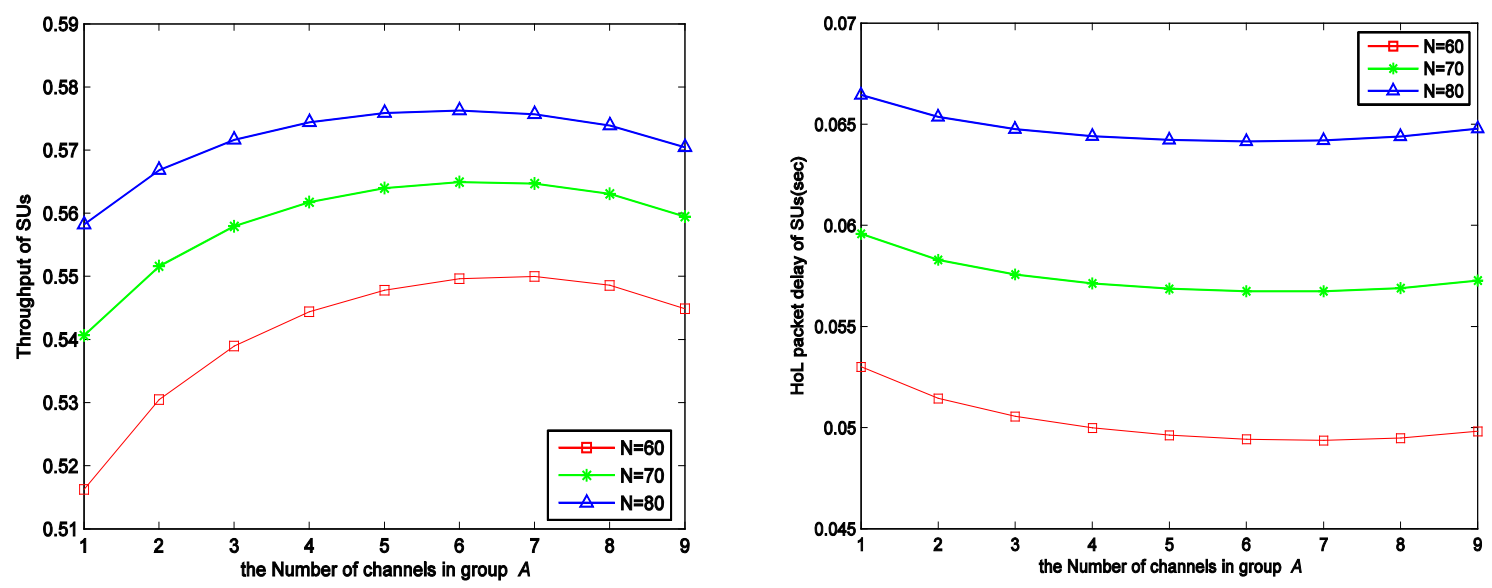

Figure 1 Performance of grouped sensing schemes versus the number of channels in group A

Figure 1 depicts the throughput of SUs and HoL packet delay of SUs in grouped sensing scheme as the number of channels in group $A$ increases and the number $N$ of SUs is 60,70 and 80 . Figure 1 shows that the throughput(or HoL packet delay) of SUs increases(or decreases) and then decreases(or increases) as the $m$ increases. We can find the optimal number $m$ to maximize the throughput of SUs. For example, when $M=10, m=7$ is the optimal number of channels in group $A$ which maximizes throughput of SUs.

Figure 2 depicts the throughput of SUs and HoL packet delay of SUs in three sensing schemes versus the number $N$ of SUs when $M=10, m=4$. Both the throughput $T_{s}$ of SUs and the HoL packet delay of SUs increase as the parameter $N$ increases. As expected, figure 2 show that the performance of SUs in grouped sensing scheme higher than that in random $m$-sensing scheme and lower than that in all sensing, because if a SU dose not find any idle channels in group $A$, then the SU will sense channels from group $B$ after its random backoff time, so it leads to more chances to find idle channels. 

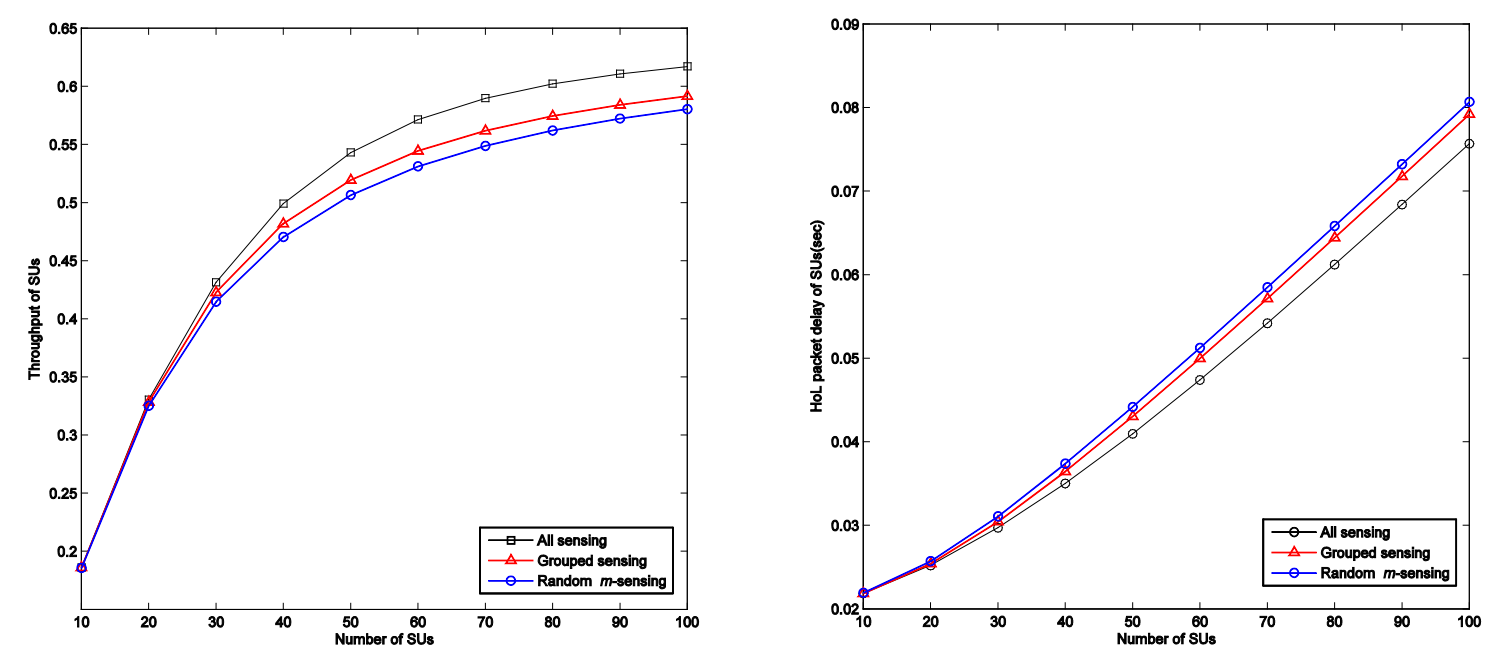

Figure 2 Performance of three sensing schemes versus the number of SUs

\section{Conclusions}

In this paper, we propose an unslotted CSMA with grouped sensing in multi-channel cognitive radio networks, and model the system by 3-demensional continuous time Markov process and provide the performance measure of the system. Numerical results show that the grouped sensing scheme overcomes the defect of low throughput in random $m$-sensing.

\section{Acknowledgements}

This research is supported by the Jilin Provincial Department of Education Science and Technology Research Project ([2015] -37), Jilin, China.

\section{References}

[1] FCC Spectrum Policy Task Force. FCC Report of the Spectrum Efficiency Working Groups. 2002, 9

[2] H. Lee, D. H. Cho. VoIP capacity analysis in cognitive radio system. Communications Letters, IEEE. 2009, 6(13): 393 395

[3] W. Eric, M.Wang, C. H. Foh. Analysis of Cognitive Radio Spectrum Access with Finite User Population. Communications Letters, IEEE. 2009, 5(13): 294 296

[4] Su Hang, Zhang Xi, “ Cross-Layer Based Opportunistic MAC Protocols for QoS Provisionings Over Cognitive Radio Wireless Networks ". IEEE Journal on Selected Areas in Communications. 26(1), 118 - 129 (Jan 2008)

[5] JW Chong, Y Sung, DK Sung. RawPEACH:Multiband CSMA/CA-Based Cognitive Radio Networks” . Journal of Communications and Networks,11(2), 174 - 185 (April 2009)

[6] Amr A. El-Sherif, Amr Mohamed."Decentralized Throughput Maximization in Cognitive Radio Wireless Mesh Networks“, IEEE TRANSACTIONS ON MOBILE COMPUTING, VOL. 13(9), , SEPTEMBER 2014:1967 1980

[7] S. H. Huang, X. Liu, Z. Ding. Opportunistic Spectrum Access in Cognitive Radio Networks. INFOCOM 2008. The 27th Conference on Computer Communications.IEEE. Phoenix, USA, 2008: 2101 2109 
[8] D.B.Zhu, J. S. Park and B.D.Choi. Performance Analysis of unslotted CSMA in the Multi-Channel Cognitive Radio Networks. QTNA2010. $5^{\text {th }}$ International Conferences on Queueing Theory and its Applications. Beijing, China, 2010: 156 161

[9] Dongbi Zhu and Huimin Wang. Performance Analysis of Unslotted CSMA with Hybrid Sensing in Multi-Channel Cognitive Radio Network. Advances in Automation and Applied Information Technologies 2013.10: 624 628

[10] D. B. Zhu and B. D. Choi. Performance Analysis of CSMA in an unslotted Cognitive Radio Network with Licensed Channels and unlicensed Channels. EURASIP Journal on Wireless Communications and Networking 2012.12 